\title{
THE SPECTRUM OF THE LAPLACIAN FOR 1-FORMS
}

\section{SHUKICHI TANNO}

ABSTRACT. Let $(M, g)$ and $\left(M^{\prime}, g^{\prime}\right)$ be compact orientable Riemannian manifolds with the same spectrum of the Laplacian for 1-forms. We prove that, for $\operatorname{dim} M=2,3,16,17, \cdots, 93,(M, g)$ is of constant curvature if and only if $\left(M^{\prime}, g^{\prime}\right)$ is so.

1. Introduction. Let $(M, g)$ be a compact orientable Riemannian manifold with metric tensor $g$. The dimension of $M$ is denoted by $m$. By $\Delta$ we denote the Laplacian acting on $p$-forms on $M$. Then we have the spectrum for each $p$ :

$$
\operatorname{Spec}^{p}(M, g)=\left\{0 \geq \lambda_{1, p} \geq \lambda_{2, p} \geq \cdots>-\infty\right\}
$$

where each eigenvalue is repeated as many times as its multiplicity indicates. One of the problems on spectra is as follows: Let $(M, g)$ and $\left(M^{\prime}, g^{\prime}\right)$ be compact orientable Riemannian manifolds with $\operatorname{Spec}^{0}(M, g)=\operatorname{Spec}^{0}\left(M^{\prime}, g^{\prime}\right)$; then is it true that " $(M, g)$ is of constant curvature $K$ if and only if $\left(M^{\prime}, g^{\prime}\right)$ is of constant curvature $K^{\prime}=K^{\prime \prime}$ ? M. Berger [1] proved that this is true for $m=2$ and 3. The author [7] proved that this is true for $m \leq 5$ (furthermore, for $m \leq 6$ if $K>0$ ).

In this paper we study the effect of $\operatorname{Spec}^{1}(M, g)=\operatorname{Spec}^{1}\left(M^{\prime}, g^{\prime}\right)$. For this we apply Patodi's results [5] on coefficients of the MinakshisundaramPleijel-Gaffney's asymptotic expansion.

Theorem A. Let $(M, g)$ and $\left(M^{\prime}, g^{\prime}\right)$ be compact orientable Riemannian manifolds with $\operatorname{Spec}^{1}(M, g)=\operatorname{Spec}^{1}\left(M^{\prime}, g^{\prime}\right)$.

(i) For $m=2, m=3$, or $16 \leq m \leq 93,(M, g)$ is of constant curvature $K$ if and only if $\left(M^{\prime}, g^{\prime}\right)$ is of constant curvature $K^{\prime}=K$.

Received by the editors July 11, 1973.

AMS (MOS) subject classifications (1970). Primary 53C20; Secondary 53C55.

Key words and phrases. Spectrum, Laplacian, constant curvature, Weyl conformal curvature tensor, Bochner curvature tensor. 
(ii) For $m=4$, if $M$ and $M^{\prime}$ have the same Euler-Poincaré characteristic $\chi(M)=\chi\left(M^{\prime}\right)$ (or equivalently, the same 2 nd Betti number $b_{2}(M)=$ $\left.b_{2}\left(M^{\prime}\right)\right)$, then $(M, g)$ is of constant curvature $K$ if and only if $\left(M^{\prime}, g^{\prime}\right)$ is of constant curvature $K^{\prime}=K$.

(iii) For $m=3$ or $m=15,(M, g)$ is an Einstein space (of constant scalar curvature $S$ ) if and only if $\left(M^{\prime}, g^{\prime}\right)$ is an Einstein space (of constant scalar curvature $S^{\prime}=S$ ).

Corollary. The Euclidean m-sphere $\left(S^{m}, g_{0}\right), m=2,3,16, \cdots, 93$, is completely characterized by the spectrum of $\Delta$ for 1-forms.

One result of V. K Patodi [5] is as follows: $\operatorname{Spec}^{p}(M, g)=\operatorname{Spec}^{p}\left(M^{\prime}, g^{\prime}\right)$ for $p=0$ and $p=1$ imply (i) of Theorem $A$ for every $m$.

$A$ theorem similar to Theorem $A$ is also true in the Kählerian case for $16 \leq$ real $\operatorname{dim} M \leq 102$ (Theorem $C$ in $\$ 5$ ).

Theorem B. Let $(M, g)$ and $\left(M^{\prime}, g^{\prime}\right)$ be compact orientable Riemannian manifolds of 4-dimension with $\operatorname{Spec}^{1}(M, g)=\operatorname{Spec}^{1}\left(M^{\prime}, g^{\prime}\right)$. If $\left(M^{\prime}, g^{\prime}\right)$ is an Einstein space, we have $\chi(M) \geq \chi\left(M^{\prime}\right)$, or equivalently, $b_{2}(M) \geq b_{2}\left(M^{\prime}\right)$. The equality holds, if and only if $(M, g)$ is also an Einstein space.

It may be noticed that instead of $\operatorname{Spec}^{1}(M, g)=\operatorname{Spec}^{1}\left(M^{\prime}, g^{\prime}\right)$, we mainly use $a_{i, 1}=a_{i, 1}^{\prime}$ for $i=0,1,2$ (cf. $(2.1)-(2.4)$ ).

2. Preliminaries. By $R=\left(R_{j k l}^{i}\right), R_{1}=\left(R_{j k}\right)$ and $S$ we denote the Riemannian curvature tensor, the Ricci curvature tensor and the scalar curvature, respectively. We put $|R|^{2}=(R, R)=\left(R_{i j k l} R^{i j k l}\right)$ and $\left|R_{1}\right|^{2}=$ $\left(R_{j k} R^{j k}\right)$. Then the Minakshisundaram-Pleijel-Gaffney's formula for $\operatorname{Spec}^{1}(M, g)$ is given by

$$
\begin{aligned}
\sum_{k} \exp \left(\lambda_{k, 1} t\right)= & (4 \pi t)^{-m / 2}\left[a_{0,1}+a_{1,1} t+\cdots+a_{N, 1} t^{N}\right] \\
& +O\left(t^{N-m / 2+1}\right), \quad t \downarrow 0,
\end{aligned}
$$

where (cf. V. K. Patodi [5]) (for $m \geq 2$ )

$$
\begin{gathered}
a_{0,1}=m \operatorname{Vol} M=m \int d M, \\
a_{1,1}=\frac{m-6}{6} \int S d M \\
a_{2,1}=\frac{1}{360} \int\left[2(m-15)|R|^{2}+2(90-m)\left|R_{1}\right|^{2}+5(m-12) S^{2}\right] d M
\end{gathered}
$$


The Weyl conformal curvature tensor $C=\left(C_{j k l}^{i}\right), C_{i j k l}=g_{i r} C_{j k l}^{r}$, is

$$
\begin{aligned}
C_{i j k l}= & R_{i j k l}-a\left(R_{j k} g_{i l}-R_{j l} g_{i k}+g_{j k} R_{i l}-g_{j l} R_{i k}\right) \\
& +b\left(g_{j k} g_{i l}-g_{j l} g_{i k}\right) S,
\end{aligned}
$$

where $a=1 /(m-2)$ and $b=1 /(m-1)(m-2)$. Then $|C|^{2}=(C, C)$ is given by

$$
|C|^{2}=|R|^{2}-4\left|R_{1}\right|^{2 /(m-2)+2 S^{2} /(m-1)(m-2) .}
$$

Then (2.4) (for $m \geq 3$ ) is rewritten as

$$
\begin{array}{r}
a_{2,1}=\frac{1}{360} \int\left[2(m-15)|C|^{2}+\left(\frac{8(m-15)}{m-2}+2(90-m)\right)|G|^{2}\right. \\
\left.+\left(5(m-12)+\frac{2(90-m)}{m}+\frac{4(m-15)}{m(m-1)}\right) S^{2}\right] d M
\end{array}
$$

where $G=\left(G_{j k}\right), G_{j k}=R_{j k}-(S / m) g_{j k} \cdot|G|^{2}=0$ holds on $M$ if and only if $(M, g)$ is an Einstein space.

Remark. If $\operatorname{Spec}^{1}(M, g)=\operatorname{Spec}^{1}\left(M^{\prime}, g^{\prime}\right)$,

(1) (2.1) implies $\operatorname{dim} M=m=m^{\prime}=\operatorname{dim} M^{\prime}$;

(2) (2.2) implies Vol $M=\operatorname{Vol} M^{\prime}$;

(3) the first Betti number $b_{1}(M)=b_{1}\left(M^{\prime}\right)$, since $b_{1}(M)$ is the multiplicity of 0 in $\operatorname{Spec}^{1}(M, g)$.

3. Proof of Theorem A. The case $m=2$. Since $|R|^{2}=S^{2}$ and $\left|R_{1}\right|^{2}=$ $S^{2} / 2$, we have $30 a a_{, 1}=\int S^{2} d M$. Hence this case reduces to $\operatorname{Spec}^{0}(M, g)$ case (cf. [1] or [2]).

The case $m=3$. Since $|C|^{2}=0,(2.7)$ is $360 a_{2,1}=\int\left[78|G|^{2}+5 S^{2}\right] d M$. Assume that $\left(M^{\prime}, g^{\prime}\right)$ is of constant curvature $K^{\prime}$. Vol $M=\operatorname{Vol} M^{\prime}$ and $\int S d M=\int S^{\prime} d M^{\prime}$ imply $\int S^{2} d M \geq \int S^{\prime 2} d M^{\prime}$, where the equality holds if and only if $S=S^{\prime}=$ constant (by Schwarz inequality). Since $\left|G^{\prime}\right|^{2}=0, a_{2,1}=$ $a_{2,1}^{\prime}$ is

$$
\int\left[78|G|^{2}+5 S^{2}\right] d M=\int 5 S^{\prime 2} d M^{\prime}
$$

This proves $|G|^{2}=0$ and $S=S^{\prime}=$ constant. Hence, $(M, g)$ is an Einstein space. Because $m=3$ (or $C=0$ ), $\left(M, g\right.$ ) is of constant curvature $K=K^{\prime}$.

The case $16 \leq m \leq 93$. The coefficient of $|G|^{2}$ in (2.7) is positive for $3 \leq m \leq 93$. Then it is easy to verify that all coefficients of $|C|^{2},|G|^{2}$ and $S^{\overline{2}}$ are positive for $16 \leq m \leq 93$. Assume that $\left(M^{\prime}, g^{\prime}\right)$ is of constant curvature $K^{\prime}$. Then $\left|C^{\prime}\right|^{2}=\left|G^{\prime}\right|^{2}=0$. As before we have $\int S^{2} d M \geq \int S^{\prime 2} d M^{\prime}$. 
Therefore $a_{2,1}=a_{2,1}^{\prime}$ implies $|C|^{2}=|G|^{2}=0$. That is, $(M, g)$ is of constant curvature $K=K^{\prime}$.

The case $m=4$. The Euler-Poincaré characteristic $\chi(M)$ of $M$ is

$$
\chi^{(M)}=\frac{1}{32 \pi^{2}} \int\left[|R|^{2}-\left.4|R|_{1}\right|^{2}+S^{2}\right] d M .
$$

Eliminating $|R|^{2}$ from (2.4) and (3.1) we obtain

$$
180 a_{2,1}+352 \pi^{2} \chi(M)=\int\left[42\left|R_{1}\right|^{2}-9 S^{2}\right] d M=\int\left[42|G|^{2}+(3 / 2) S^{2}\right] d M
$$

Assume that $\left(M^{\prime}, g^{\prime}\right)$ is of constant curvature $K^{\prime}$. Then from $\left|G^{\prime}\right|^{2}=0$,

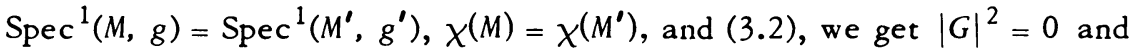
$S=S^{\prime}=$ constant. Again by (2.7) and $\left|C^{\prime}\right|^{2}=0$, we get $|C|^{2}=0$. Thus, $(M, g)$ is of constant curvature $K=K^{\prime}$. Since $\chi(M)=2-2 b_{1}(M)+b_{2}(M)$, this proves (ii).

Finally we show (iii) for $m=15$. By (2.7) we get $360 a_{2,1}=$ $\int\left[150|G|^{2}+25 S^{2}\right] d M$. Then the proof is similar to the above discussion.

4. Proof of Theorem B. By assumption we have $a_{i, 1}=a_{i, 1}^{\prime}, i=0,1$, 2 , and $\left|G^{\prime}\right|^{2}=0$. Then $\int S^{2} d M \geq \int S^{\prime 2} d M$. (3.2) gives

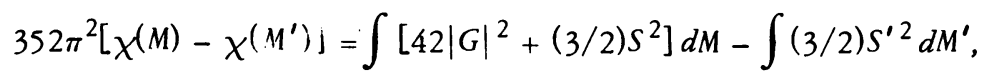

from which we obtain Theorem B.

Remark. It is interesting to see that for $\operatorname{Spec}^{0}(M, g)$ case we have $\chi^{(M)} \leq \chi^{\left(M^{\prime}\right)}$ in the similar situation (cf. S. Tanno [7]).

5. Kählerian manifolds. Let $(M, g, J)$ be a Kählerian manifold with almost complex structure tensor $J$ and Kählerian metric tensor $g$. They satisfy $J_{j}^{i} J_{k}^{j}=-\delta_{k}^{i}$ and $g_{i j} J_{r}^{i} J_{s}^{j}=g_{r s}$, and $J$ is parallel. The Bochner curvature tensor $B=\left(B_{j k l}^{i}\right), B_{i j k l}=g_{i r} B_{j k l}^{r}$, is given by

$$
\begin{aligned}
B_{i j k l}=R_{i j k l}-a^{*}\left(R_{j k} g_{i l}-R_{j l} g_{i k}+g_{j k} R_{i l}-g_{j l} R_{i k}+R_{j r} J_{k}^{r} J_{i l}\right. \\
\left.-R_{j r} J_{l}^{r} J_{i k}+J_{j k} R_{i r} J_{l}^{r}-J_{j l} R_{i r} J_{k}^{r}-2 R_{k r} J_{l}^{r} J_{i j}-2 R_{i r} J_{j}^{r} J_{k l}\right) \\
+b^{*}\left(g_{j k} g_{i l}-g_{j l} g_{i k}+J_{j k} J_{i l}-J_{j l} J_{i k}-2 J_{k l} J_{i j}\right) S,
\end{aligned}
$$

where $a^{*}=1 /(m+4), b^{*}=1 /(m+2)(m+4)$, and $|B|^{2}=(B, B)$ is given by (cf. [6], [7], etc.) 


$$
|B|^{2}=|R|^{2}-16\left|R_{1}\right|^{2} /(m+4)+8 S^{2} /(m+2)(m+4)
$$

Theorem C. Let $(M, g, J)$ and $\left(M^{\prime}, g^{\prime}, J^{\prime}\right)$ be compact Kählerian manifolds with $\operatorname{Spec}^{1}(M, g)=\operatorname{Spec}^{1}\left(M^{\prime}, g^{\prime}\right)$. For $16 \leq m=$ real $\operatorname{dim} M \leq 102$, $(M, g, J)$ is of constant holomorphic sectional curvature $H$ if and only if $\left(M^{\prime}, g^{\prime}, J^{\prime}\right)$ is of constant holomorphic sectional curvature $H^{\prime}=H$.

Proof. (2.4) is written as

$$
\begin{aligned}
a_{2,1}=\frac{1}{360} \int\left[2(m-15)|B|^{2}\right. & +\left(\frac{32(m-15)}{m+4}+2(90-m)\right)|G|^{2} \\
& \left.+\left(5(m-12)+\frac{2(90-m)}{m}+\frac{16(m-15)}{m(m+2)}\right) s^{2}\right] d M
\end{aligned}
$$

The coefficient of $|G|^{2}$ is positive for $m \leq 103$. Hence, for $16 \leq m \leq 102$, all coefficients of $|B|^{2},|G|^{2}$ and $S^{2}$ are positive. If we notice that a Kählerian manifold is of constant holomorphic sectional curvature, if and only if $|B|^{2}=|G|^{2}=0$, then we get Theorem $C$.

Corollary. The complex projective space $\left(C P^{n}, g_{0}, J_{0}\right)$ with FubiniStudy metric, $16 \leq 2 n \leq 102$, is completely characterized by the spectrum of the Laplacian for 1-forms.

\section{REFERENCES}

1. M. Berger, Le spectre des variétés riemanniennes, Rev. Roumaine Math. Pures Appl. 13(1968), 915-931. MR 39 \#892.

2. M. Berger, P. Gauduchon and E. Mazet, Le spectre d'une variété riemannienne, Lecture Notes in Math., vol. 194, Springer-Verlag, Berlin and New York, 1971. MR $43 \# 8025$.

3. M. P. Gaffney, Asymptotic distributions associated with the Laplacian for forms, Comm. Pure Appl. Math. 11(1958), 535-545. MR 20 \#580.

4. V. K. Patodi, Curvature and the eigenforms of the Laplace operator, J. Differential Geometry 5(1971), 233-249, MR 45 \# 1201.

5. - Curvature and the fundamental solution of the heat operator, J. Indian Math. Soc. 34(1970), 269-285.

6. S. Tanno, An equality for 4-dimensional Kählerian manifolds, Proc. Japan Acad. 49(1973), 257-261.

7. ——, Eigenvalues of the Laplacian of Riemannian manifolds, Tôhoku Math. J. 25(1973), 391-403.

MATHEM ATICAL INSTITUTE, TÔHOKU UNIVERSITY, SENDAI, JAPAN 